\title{
„Die Digitalisierung verbessert nicht die medizinische Versorgung als solche"
}

\author{
Die moderne Kommunikation macht auch vor der Arzt-Patienten-Beziehung nicht halt: Auch \\ wenn es für viele noch futuristisch klingen mag, seit dem 1. April 2017 wird die Online-Sprech- \\ stunde eingeschränkt möglich. Der GKV-Spitzenverband und die Kassenärztliche Bundesvereini- \\ gung hatten sich im Februar auf die Vergütung für Videosprechstunden verständigt. Damit \\ können nun Hausärzte und die meisten niedergelassenen Fachärzte im Rahmen einer Video- \\ sprechstunde den Verlauf bestimmter Krankheiten bei Patienten, deren letzter persönlicher Arzt- \\ kontakt nicht länger als zwei Quartale zurückliegt, per Kamera kontrollieren und beraten.
}

Auf einer Pressekonferenz von der Schwenninger Krankenkasse (SKK) zum Thema „Schnell, effizient und zum Wohle des Patienten - wieviel Digitalisierung verträgt unsere Gesundheit?“ diskutierten nun Ende März David Meinertz, Gründer und Geschäftsführer der in England basierten Online-Arztpraxis DrEd, und Dirk Heidenblut, Mitglied im Gesundheitsausschuss für Digitalisierung des Deutschen Bundestages, über Möglichkeiten und Risiken der Digitalisierung im Gesundheitswesen.

\section{Videosprechstunde: Es darf keine körperliche Untersuchung nötig sein}

So ist in Deutschland wegen des Fernbehandlungsverbots eine Online-Arztpraxis unzulässig. Was seit April hierzulande eingeschränkt möglich ist, ist bei der Online-Praxis DrEd schon seit 2011 an 200.000 Patienten allein aus Deutschland durchgeführt worden: DrEd berät und behandelt 35 Krankheitsbilder aus der Ferne. Bei unspezifischen Schmerzen verweist man die Patienten an den Arzt vor Ort - es darf also keine körperliche Untersuchung nötig sein.

„Ich sympathisiere mit allem, was Bewegung ins deutsche Gesundheitswesen bringt", sagte Dirk Heidenblut auf die Frage, was er von einer solchen Online-Praxis halte. Er hoffe, dass durch die Lockerung des berufsstandrechtlichen Fernbehandlungsverbots nun im deutschen Gesundheitswesen „etwas vorangehe“. E-Health verbessere schließlich den Zugang zur medizinischen Versorgung und die Informiertheit der Patienten. Allerdings, so betonte er: „Die Digitalisierung als solche verbessert nicht die medizinische Versorgung.“

Bisher dürfen in Deutschland Mediziner gemäß der Berufsordnung für Ärzte keine Diagnosen ohne mindestens einen direkten Patientenkontakt stellen. Seit Juni 2016 ist in BadenWürttemberg das Fernbehandlungsverbot bereits teilweise gelockert, dort darf seither auch der Erstkontakt zwischen Arzt und Patient digital erfolgen.

Hat eine Videosprechstunde auch im zahnärztlichen Bereich einen Patientennutzen? Der Bundesvorsitzende des Freien Verbandes Deutscher Zahnärzte, Harald Schrader, sagt hierzu Nein: „Voraussetzung für die zahnärztliche Behandlung ist in erster Linie eine eingehende Inspektion der Mundhöhle inklusive einer Sondierung der Zähne und Palpation der Weichteilstrukturen. Auch Kontrollen nach Behandlungen sind nur durch direkten Augenschein möglich.“

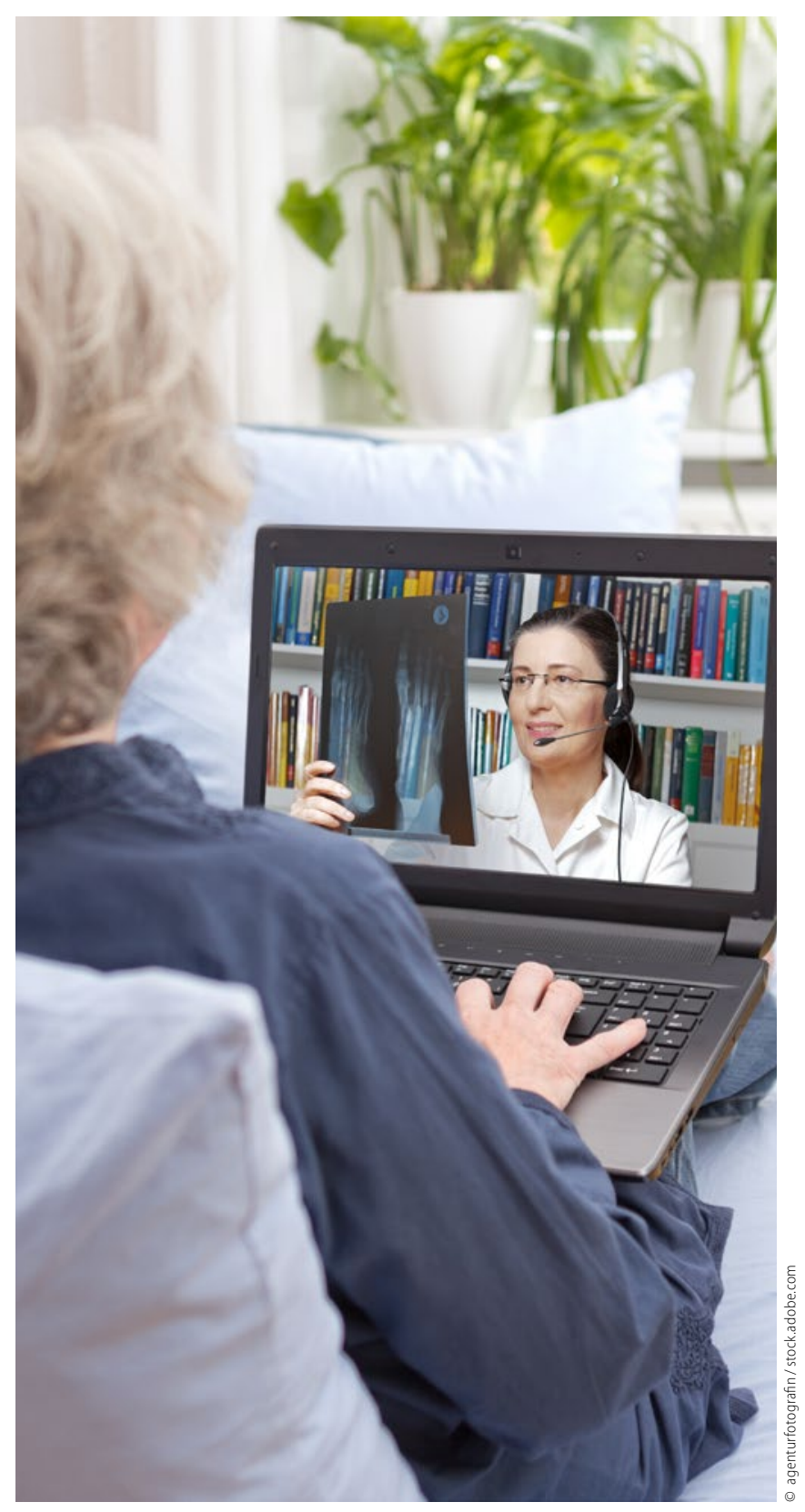

